\title{
Circulatory Response to Passive and Active Changes in Posture
}

\author{
T Heldt $^{1}$, MB Oefinger ${ }^{2}$, M Hoshiyama $^{1}$, RG Mark ${ }^{1,2}$ \\ ${ }^{1}$ Harvard University - MIT Division of Health Sciences and Technology \\ ${ }^{2}$ Department of Electrical Engineering and Computer Science \\ Massachusetts Institute of Technology, USA
}

\begin{abstract}
To understand the sequence of hemodynamic events elicited by active (stand-up) and passive (head-up tilt, HUT) changes in posture, we monitored heart rate and arterial blood pressure continuously in ten healthy volunteers at rest, during rapid tilts $\left(75^{\circ}\right.$ HUT over 2 secs), slow tilts $\left(75^{\circ}\right.$ HUT over 50 secs), and stand tests. A marked initial transient drop in mean arterial pressure (MAP) and increase in heart rate $(H R)$ seen during rapid tilt, $R T$, and stand-up, SU, were absent during slow tilt, ST. While the magnitudes of transient heart rate peak and blood pressure minimum for $R T$ and $S U$, respectively, were different, their relative timing was well preserved. We attribute the timing to the response of homeostatic reflex mechanisms. No statistically significant difference could be detected when comparing steady-state values of MAP and HR for $S U$ and HUT 140 seconds after the onset of the respective stress.
\end{abstract}

\section{Introduction}

Cardiovascular adaptation to the upright posture depends on the proper interplay of the hemodynamic system and the reflex mechanisms that maintain blood pressure homeostasis. Failure of one of the subsystems to adapt to the gravitation-induced shifts in blood volume often results in a collection of clinical symptoms termed orthostatic intolerance (OI).

Clinically, OI is diagnosed on the basis of a drop in arterial blood pressure or an excessive increase in heart rate measured a certain time after the assumption of the upright posture. However, little is learned about the mechanisms underlying OI by making a single isolated measurements of MAP and HR at a certain time after onset of gravitational stress. Research done in our laboratory aims at using the information contained in the transient response of hemodynamic variables to gravitational stress to identify mechanisms underlying cardiovascular maladaptation to the upright posture. To achieve this goal, it is necessary to understand better the transient hemodynamic events elicited by an active change in posture and passive changes in posture. Specifically, we are interested in quantifying the degree of similarity between the hemodynamic responses seen during stand up, rapid, and slow head-up tilt.

\section{Methods}

We recruited ten healthy volunteers ( 5 males, 5 females) to participate in this study. The mean age was $28.7 \pm 1.2$ years, the mean height was $172.8 \pm 4.0 \mathrm{~cm}$, and the mean weight was $70.6 \pm 4.5 \mathrm{~kg}$. Participants regularly engaged in light to moderate physical activity and had no sign of cardiovascular disease. Each volunteer gave written, informed consent prior to participation in the study. Each participant was instrumented with a standard clinical ECG monitor and a non-invasive blood pressure monitoring device (FINAPRES). ECG and the arterial pressure waveforms were recorded throughout the duration of the experiment. After instrumentation, subjects rested on a tilt table with foot support and subsequently underwent a series of six changes in posture: two stand-ups, two rapid HUTs $\left(75^{\circ}\right.$ HUT over 2 secs), and two slow HUTs ( $75^{\circ}$ HUT over 50 secs) each lasting for three minutes and separated by five minutes in the resting supine position. At the conclusion of the final intervention, the subject was returned to the horizontal position for a final five minutes of supine baseline recording. The sequence of six interventions was randomized for each subject. The study protocol was approved by MIT's Committee On the Use of Humans as Experimental Subjects and the Advisory Board of the MIT-MGH General Clinical Research Center. The study was carried out at the MIT General Clinical Research Center. In the data processing step, we used a curve length-based QRS detection algorithm [1] to detect the onset of ventricular contractions and a slope-based detection algorithm [2] to identity the arterial pressure wave onsets. Time series for systolic arterial pressure, SAP, mean arterial pressure, MAP, diastolic arterial pressure, DAP, and heart rate, HR, were generated through linear interpolation between the respective beat-by-beat values.

\footnotetext{
${ }^{1}$ Throughout this paper data are presented as population mean \pm standard error of the mean.
} 
Table 1. Steady-state numerics

\begin{tabular}{|c|c|c|c|}
\hline & Rest & $\overline{\mathrm{HU}}$ & $\Delta$ \\
\hline \multicolumn{4}{|c|}{ Heart rate (beats/min) } \\
\hline RT & $67.2 \pm 2.4$ & $80.1 \pm 2.1^{*}$ & $13.0 \pm 1.5$ \\
\hline ST & $66.5 \pm 2.4$ & $81.1 \pm 2.2^{*}$ & $14.6 \pm 1.8$ \\
\hline SU & $65.1 \pm 2.6^{\dagger}$ & $79.0 \pm 2.2^{*}$ & $13.9 \pm 1.5$ \\
\hline \multicolumn{4}{|c|}{ Systolic arterial pressure $(\mathrm{mm} \mathrm{Hg})$} \\
\hline RT & $108.5 \pm 3.5$ & $106.6 \pm 3.8$ & $-1.9 \pm 1.7$ \\
\hline ST & $108.4 \pm 2.9$ & $108.8 \pm 2.9^{\dagger}$ & $0.4 \pm 2.2$ \\
\hline SU & $109.8 \pm 3.2$ & $111.5 \pm 3.7$ & $1.7 \pm 1.9$ \\
\hline \multicolumn{4}{|c|}{ Mean arterial pressure $(\mathrm{mm} \mathrm{Hg})$} \\
\hline RT & $69.3 \pm 2.1$ & $73.3 \pm 2.6^{*}$ & $4.0 \pm 1.2$ \\
\hline ST & $70.7 \pm 1.8$ & $75.5 \pm 2.1^{*}$ & $5.7 \pm 1.4$ \\
\hline SU & $70.5 \pm 2.1$ & $75.7 \pm 2.6^{*}$ & $5.2 \pm 1.4$ \\
\hline \multicolumn{4}{|c|}{ Diastolic arterial pressure $(\mathrm{mm} \mathrm{Hg})$} \\
\hline RT & $54.8 \pm 1.9$ & $60.9 \pm 2.6^{*}$ & $6.3 \pm 1.2$ \\
\hline ST & $56.3 \pm 1.8$ & $63.0 \pm 2.2^{*}$ & $6.8 \pm 1.2$ \\
\hline SU & $55.6 \pm 1.9$ & $62.5 \pm 2.5^{*}$ & $6.9 \pm 1.3$ \\
\hline
\end{tabular}

\section{Results}

\subsection{Steady-state numerics}

In Table 1, we list the average values of HR, SAP, MAP, and DAP $50 \mathrm{sec}$ before (Rest) and $140 \mathrm{sec}$ after assumption of the head-up (HU) position along with the difference between the two $(\Delta)$ for RT, ST, and SU, respectively. Statistical comparisons are based on two-sided tests of significance for comparisons between different interventions (e.g., comparison of resting HR between RT and $\mathrm{SU}$ ) and one-sided tests of significance for $\mathrm{HU}$ vs. Rest comparisons. Each intervention led to statistically significant $(P<0.005)$ increases in $H R$, MAP, and DAP. Neither intervention led to a statistically significant change in SAP. With two exceptions, no statistically significant difference was observed between the resting steady-state hemodynamic variables for the three interventions or between the head-up steady-state hemodynamic variables for the three interventions.

\subsection{Transient responses}

The following three figures show the populationaveraged hemodynamic responses (solid lines) along with the 95\% approximate confidence intervals (shaded gray areas) of MAP and HR for SU (Figure 1), RT (Figure 2), and ST (Figure 3). Responses are shown from 60 seconds before to 180 seconds after the onset of gravitational stress. To emphasize the dynamic response, we have removed the respective resting values. Also note that in order to accommodate the large transient drop in MAP and increase

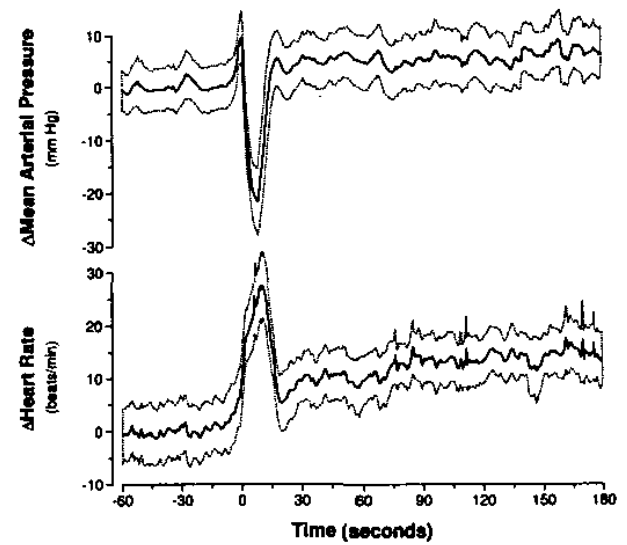

Figure 1: Hemodynamic response to standing up.

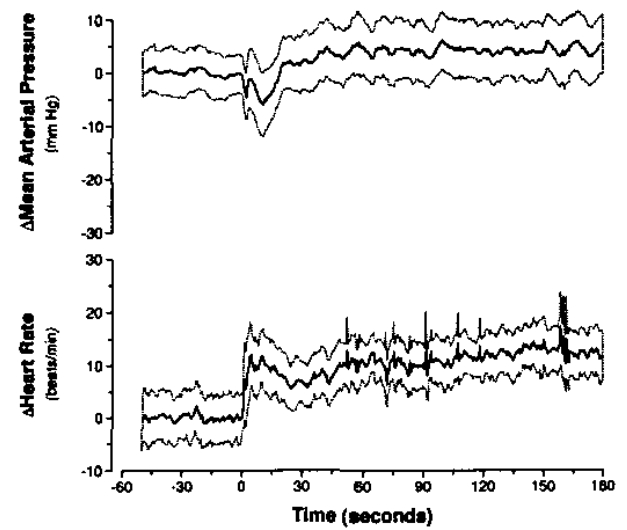

Figure 2. Hemodynamic response to rapid HUT.

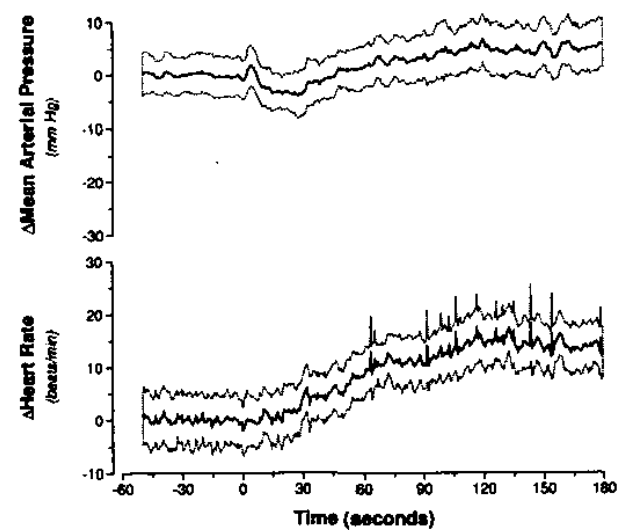

Figure 3. Hemodynamic response to slow HUT. 


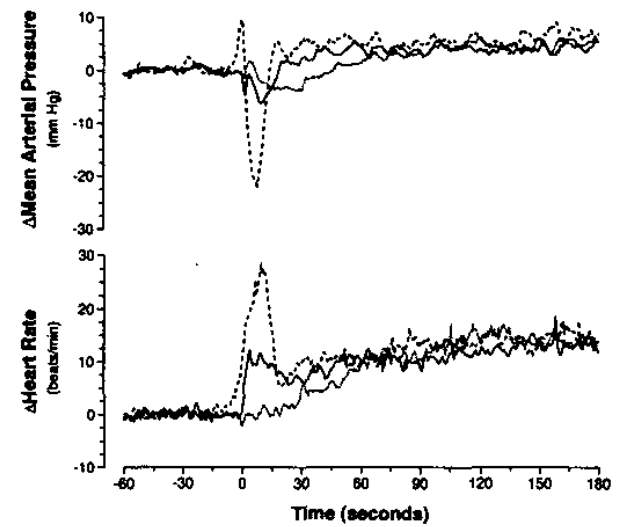

Figure 4. Comparison of transient responses for SU (dashed lines), RT (solid lines), and ST (dotted lines).

in HR, the abscissas in Figure 1 have been rescaled. It is evident from Figures 1-3 that each intervention leads to a somewhat different transient response. Clearly SU elicits the most dramatic transient response in MAP and HR. The MAP response to $S U$ is characterized by a brief increase followed by a dramatic decrease in blood pressure. The former being due to a Valsalva maneuver that allows for raising of the upper body off the tilt table. HR transiently increases dramatically in response to the drop in arterial pressure. During RT, the subjects are tilted passively with minimal muscular effort on their part. It is therefore not surprising, that the initial Valsalva-related blood pressure rise prior to the change in posture seen during $S U$ is absent during RT. However, the blood pressure response to RT does show a bi-phasic behavior: a drop in MAP is followed by an increase which in turn is followed by an even larger decrease. Whether the intermittent increase in MAP is caused by a transient increase in cardiac output [3] or due to reflexive muscle contraction in response to the rapid tilt requires further elucidation. The HR response to RT shows a transient increase similar in duration to the one seen during SU. The cardiovascular response to ST is characterized by very gradual changes in both HR and MAP. Interestingly, a small increase in MAP is also seen at the onset of ST.

In Figure 4, we directly compare the dynamic responses of MAP and HR of SU (dashed lines), RT (solid lines), and ST (dotted lines). Comparing RT and SU, clear and statistically significant differences exist in the amplitudes of the initial blood pressure drop and heart rate increase. Yet the timing of the initial transient responses for these two interventions is well preserved, i.e. differences are confined to the first 20 seconds of the transient response. In contrast, the hemodynamic response to ST lacks completely the initial transient complex seen during SU and RT.

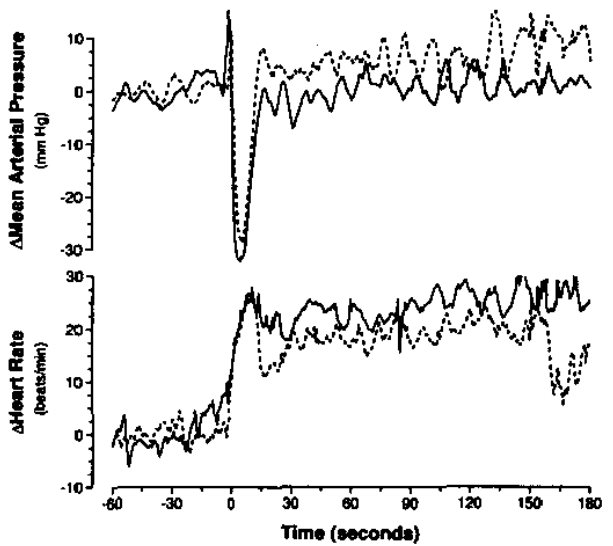

Figure 5. Comparison of transient responses for SU (dashed lines) and RT (solid lines) with prior muscle contraction.

\section{Pilot study}

In a follow-up pilot study, we investigated the origin of the difference in the amplitude response seen between SU and RT. One obvious mechanistic difference between the two interventions is the contraction of abdominal and leg muscles in the process of standing up. We hypothesized that the transient response to standing can be mimicked by a rapid tilt with prior muscle contraction. To test this hypothesis, we conducted a pilot study in which two volunteers underwent a protocol similar to the one outlined above. We substituted for the slow tilts a modified rapid tilt in which the participants were asked to lift their legs off the tilt table for about two seconds just prior to being tilted up rapidly. The results of this pilot study are shown in Figure 5 and are based on two volunteers with a total of six rapid tilts with abdominal contraction (solid lines) and six stand tests (dashed lines). It is obvious from Figure 5 that muscular contraction just prior to rapid tilting gives rise to transient changes in MAP and HR that are very similar, if not indistinguishable, from those elicited by actively standing up.

\section{Discussion}

This study was designed to elucidate the short-term $(\leq$ 3 mins), transient hemodynamic response to the upright posture for three different modes of transition, namely rapid tilt, slow tilt, and standing-up. As can be seen from Table 1, the steady-state responses of MAP, DAP, and HR are independent of the mode of transition to the head-up position. It seems physiologically intuitive that the steady-state response depends only on the amount of blood volume that is translocated to the dependent 
vasculature. The results in Table 1 indicate that the mode of transition is irrelevant if one is only interested in the steadystate cardiovascular response. Significant differences exist, however, between the three modes of transition during the first 20-30 seconds. As mentioned above, the timing of the initial transient complex during SU and RT is well preserved. In both interventions, the gravitational stress is brought on rapidly and can be considered a quasi stepfunctional change in gravitational stress. Rapid vagal withdrawal will be the body's first line of defense leading to a rapid, almost immediate increase in heart rate. The sympathetic system is known to have longer latency and impulse response and will therefore respond slower to the gravitational stimulus. We attribute the fact that the hemodynamic response to $\mathrm{SU}$ and $\mathrm{RT}$ is essentially the same after $\approx 20$ seconds to the intrinsic equilibration of sympathetic outflow and vagal withdrawal. Further slow increases is heart rate are seen for both SU and ST and are probably related to extravasation of plasma volume into the interstitial fluid compartment. Cardiovascular adaptation to ST exhibits a more gradual change in both $\mathrm{HR}$ and MAP lacking the initial transient complex seen during both RT and SU. We attribute this behavior to the slow change in gravitational stress which essentially allows for equilibration of the reflex responses during transition to the head-up posture. This explanation is supported by the fact that the hemodynamic response to ST becomes indistinguishable from those seen during RT and $\mathrm{SU}$ about 50 seconds after the onset of gravitational stress which is the time it takes for the slow tilt to be completed.

Finally, a small pilot study suggests that the difference between the hemodynamic responses seen during RT and SU can be explained on the basis of muscle contraction. Whether the large drop in MAP and the reflexive increase in $H R$ are mediated through peripheral vasodilation as suggested by some $[4,5]$ or whether mechanical factors such as transient changes in venous return due to abdominal contraction play a dominant role remains to be elucidated.

\section{Acknowledgments}

This work was partially supported by NASA through the NASA Cooperative Agreement NCC 9-58 with the National Space Biomedical Research Institute and through NIH grant M01-RR01066 to the General Clinical Research Center at MIT.

\section{References}

[1] Zong W, Moody GB, Jiang D. A robust open-source algorithm to detect onset and duration of $\mathrm{QRS}$ complexes. Comput Cardiol 2003; 30.

[2] Zong W, Heldt T, Moody GB. An open-source algorithm to detect onset of arterial blood pressure pulses. Computers in Cardiology 2003; 30 .
[3] Toska $K$ and Walløe. The dynamic time course of hemodynamic responses after passive head-up tilt and tiltback to a supine position in healthy humans. J Appl Physiol 2002;92(4): 1671-6.

[4] Tanaka H, Sjöberg BJ, Thulesius O. Cardiac output and blood pressure during active and passive standing. Clin Physiol 1996; 16: 157-170.

[5] Sprangers RLH, Wesseling KH, Imholz ALT, Imholz BPM, Wieling W. Initial blood pressure fall on stand up and exercise explained by changes in total peripheral resistance. J Appl Physiol 1991; 70(2):523-530.

Address for correspondence:

Roger G. Mark, MD, PhD

Massachusetts Institute of Technology

E25-505 / Cambridge, MA 02139 / USA

rgmark@mit.edu 